\title{
Uma Versão Geométrica do Teorema de Vincent
}

Vincent's theorem from the geometrical viewpoint

Jean Fernandes Barros'

\section{Resumo}

Neste trabalho, apresentamos uma versão geométrica do teorema de Vincent, conforme Alesina e Galuzzi (2000). A versão clássica, como em Vincent (1836); Akritas (1981); Alesina e Galuzzi (1998), é dada em termos de frações contínuas, e tem por finalidade a obtenção de uma solução númerica para as equações algébricas. Para uma demonstração da versão geométrica do teorema de Vincent, nós utilizamos os resultados de Ostrowski (1950).

Palavras-chave: Teorema de Vincent; Regra de Sinais de Descartes; Transformações de Möbius

\section{Abstract}

In this work, we present a geometrical version of Vincent's theorem, and according with Alesina e Galuzzi (2000). The classical version, as seen in Vincent (1836); Akritas (1981); Alesina e Galuzzi (1998), uses continued fractions, whose goal is to obtain numerical solutions for the algebraic equations. For a proof of the geometrical version of Vincent's theorem, we utilize Ostrowski's results, as in Ostrowski (1950).

Keywords: Vincent's theorem; Descartes's rule of signs; Möbius transformations 


\section{Introdução}

O teorema de Vincent fornece um método para a separação dos zeros de um polinômio numa variável, como constatamos em Vincent (1836); Akritas (1981); Alesina e Galuzzi (1998); Uspensky (1948). E também, com o auxílio da regra de sinais de Descartes, o teorema de Vincent inspira um método para contar classes de configurações centrais em Mecânica Celeste. Para maiores detalhes, consultem Barros e Leandro $(2011,2014)$ e Leandro (2003, 2006).

Aqui, exibimos uma versão geométrica deste teorema, ver seção 4 . A versão clássica que encontramos em Akritas (1981), utiliza a teoria de frações contínuas, ver seção 3. Uma abordagem mais moderna, vista em Alesina e Galuzzi (1998), também utiliza esta teoria, porém já inicia de uma forma incipiente quando comparada com a versão em Alesina e Galuzzi (2000), uma exploração das propriedades das transformações de Möbius. Na realidade, em Vincent (1836), já se percebe a utilização das transformações de Möbius, pois Vincent inspirou-se num resultado de Lagrange (1808), em que se observa a utilidade destas transformações. É verdade que as transformações de Möbius surgem de forma natural, quando observarmos os convergentes de uma fração contínua, já que os convergentes são composições de transformações de Möbius, que ainda é uma transformação de Möbius.

Nas versões mencionadas, consideram-se os polinômios sem zeros múltiplos, o que não acarreta prejuízos aos nossos interesses. O leitor pode encontrar em Alesina e Galuzzi (1998) uma versão para zeros múltiplos, em que se utiliza extensões da regra de sinais de Descartes, que incluem os zeros complexos, como observa-se em Obreschkoff $(1963,2003)$. Além disso, em Uspensky (1948) existe um procedimento para reduzir o caso em que os zeros são múltiplos àqueles em que os zeros são simples.

Os fatos históricos relacionados ao teorema de Vincent constituem um episódio a parte da história da Matemática. A primeira publicação aparece em $1834 \mathrm{em}$ Mémories de La Société Royale de Lille. Permaneceu esquecido até 1948, quando Uspensky (1948), utilizando-o, descreveu um método completo para separar zeros de um polinômio numa variável. Neste ponto, surge uma controvérsia, já que Akritas (1986) mostra que não existe um método de Uspensky. O mérito de Uspensky foi reviver o método de Vincent. Posteriormente, o teorema aparece num livro de Obreschkoff (1963). Depois disto, ganha notoriedade com os trabalhos de Akritas, que obtém um algoritmo bastante eficiente de separação dos zeros de um polinômio numa variável, desbancando o que se encontra em Uspensky (1948) e o método de Sturm. Relatos históricos mostram que o esquecimento vivido por Vincent foi devido à popularidade alcançada pelo método de Sturm. Para mais detalhes, pode-se consultar Akritas e Danielopoulos (1978); Akritas (1978, 1981, 1986); Alesina e Galuzzi (1998).

Bem, nossa contribuição é dar uma demonstração do teorema de Vincent, da versão fornecida por Alesina e Galuzzi (2000), através dos resultados de Ostrowski (1950), ver seção 4.

\section{Descartes, Möbius e Lagrange}

Para o que se segue, necessitamos da seguinte

Definição 2.1. Dada uma sequência, finita ou infinita, de números reais

$$
a_{0}, a_{1}, a_{2}, \ldots,
$$

dizemos que existe uma variação de sinal (uma permanência de sinal) entre dois termos $a_{t}$ e $a_{s}$, digamos, com $t<s$, se uma das seguintes condições ocorre:

1. $s=t+1$ e os termos $a_{t}$ e $a_{s}$ têm sinais opostos (têm o mesmo sinal);

2. $s>t+1$ e os termos $a_{t+1}, a_{t+2}, \ldots, a_{s-1}$ são todos zeros, enquanto $a_{t}$ e $a_{s}$ têm sinais opostos (têm mesmo sinal).

No caso de uma sequência finita de comprimento $n+1$, digamos, $a_{0}, a_{1}, \ldots, a_{n}$, o número de variações de sinais (permanências de sinais) é o número de pares $(t, s)$ satisfazendo as condições (1) ou (2).

Observação 2.1. Seja $P \in \mathbb{R}[x]$, digamos, $P=\sum_{i=0}^{n} a_{i} x^{i}$, com $a_{n} \neq 0$, isto é, $P$ tem grau $n$. Observemos que os coeficientes de $P$ formam uma sequência finita, também denotada por $P$, de comprimento $n+1$. Denotaremos $V(P)$ o número de variações de sinais de $P$. E, também, denotaremos por $\mathcal{P}(P)$ o número de permanências de $P$. Observemos que $V(P) \leq n$ e $\mathcal{P}(P) \leq n$.

Exemplo 2.2. Seja o polinômio $P(x)=-2 x^{9}-5 x^{7}+$ $x^{4}+7 x^{3}-x+1$. Considerando a sequência dos seus coeficientes, $-2,0,-5,0,0,1,7,0,-1,1$, temos que $V(P)=$ 3 e $\mathcal{P}(P)=2$.

Em La Géométrie, Descartes (1637) afirmou: “Uma equacão algébrica tem tantas raízes verdadeiras (isto é, positivas) quantas são as mudanças de sinais de + para - ou de - para +. E tantas raízes falsas (isto é, negativas) quantos são o número de vezes que dois sinais positivos ou dois sinais negativos aparecem em sucessão".

A afirmação acerca da quantidade de raízes falsas, isto é, negativas, é válida somente para polinômios cujos termos, em ordem decrescente ou crescente, têm graus pares e ímpares que se alternam. De fato, dados dois 
termos consecutivos, um tem grau par, e, o outro, grau ímpar. Desta forma, se houver uma permanência de sinais, quando trocamos $x$ por $-x$, o termo de grau par permanece com o mesmo sinal, enquanto o termo de grau ímpar muda de sinal. Isto significa que passamos a ter uma variação de sinal. Em particular, os polinômios completos, isto é, os polinômios que possuem todos os seus coeficientes não-nulos, são polinômios deste tipo. Por exemplo, considerando o polinômio $P(x)=x^{8}-$ $x^{7}-x^{5}-x^{3}-x+1$, temos que $V(P)=2$ e $\mathcal{P}(P)=3$. Agora, para $Q(x)=P(-x)=x^{8}+x^{7}+x^{5}+x^{3}+x+1$, temos que $V(Q)=0$ e $\mathcal{P}(Q)=5$.

A seguir, vemos um enunciado da regra de sinais de Descartes, onde $Z(P)$ representa o número de zeros positivos de $P$, contados com multiplicidades. Uma demonstração pode ser vista em Wang (2004). Uma outra demonstração pode ser vista em Uspensky (1948).

Teorema 2.3. (Regra de Sinais de Descartes) Seja $P(x):=$ $a_{n} x^{b_{n}}+\ldots+a_{1} x^{b_{1}}+a_{0} x^{b_{0}}$ um polinômio real cujos coeficientes são não-nulos, onde $b_{i} \in \mathbb{Z}$, para $i=0,1, \ldots, n$, são tais que $0 \leq b_{0}<b_{1}<\cdots<b_{n}$. Então,

$$
Z(P) \leq V(P) \text { e } V(P) \equiv_{2} Z(P),
$$

isto é, $V(P)$ e $Z(P)$ têm a mesma paridade.

Existem três casos em que a regra de sinais de Descartes conta exatamente o número de zeros positivos. Primeiro, o caso em que $V(P)=0$. Neste caso, $Z(P)=0$. Vê-se, em geral, que não temos a recíproca disto, como mostra-nos o exemplo

$$
P(x)=\left(x-\frac{1}{2}-i \frac{\sqrt{3}}{2}\right)\left(x-\frac{1}{2}+i \frac{\sqrt{3}}{2}\right)=x^{2}-x+1 .
$$

Segundo, o caso em que $V(P)=1$. Neste caso, como $V(P) \equiv_{2} Z(P)$, temos que $Z(P)=1$. Aqui, também, em geral, não vale a recíproca, já que

$$
P(x)=(x-1)\left(x^{2}-x+1\right)=x^{3}-2 x^{2}+2 x-1 .
$$

O terceiro, é quando todos os zeros de $P$ são reais, ver Eigenwillig (2008).

Para apresentarmos a versão geométrica do teorema de Vincent, relembramos alguns resultados sobre as transformações de Möbius. Uma transformação de Möbius é uma função

$$
\phi: \mathbb{C} \backslash\left\{-\frac{d}{c}\right\} \longrightarrow \mathbb{C}
$$

da forma

$$
\phi(x ; \alpha, \beta, c, d)=\frac{\alpha x+\beta}{c x+d},
$$

onde $\alpha, \beta, c, d \in \mathbb{C}$ são tais que $\alpha d-\beta c \neq 0$.

Observemos que os coeficientes $\alpha, \beta, c$ e $d$ não são únicos, já que, dado $\delta \in \mathbb{C} \backslash\{0\}$,

$$
\phi(x ; \delta \alpha, \delta \beta, \delta c, \delta d)=\phi(x ; \alpha, \beta, c, d) .
$$

Além disso, é fácil ver que a composição de transformações de Möbius é uma transformação de Möbius, e que a inversa de uma transformação de Möbius é uma transformação de Möbius. E mais, toda transformação de Möbius é uma composição de translações, rotações (coeficientes não-reais), homotetias (coeficientes reais) e de inversões. Segue-se que toda transformação de Möbius é um difeomorfismo analítico em $\mathbb{C} \backslash\left\{-\frac{d}{c}\right\}$. As demonstrações destes fatos podem ser encontradas em Ahlfors (1949).

Como o nosso caso de interesse é aquele em que os coeficientes são reais, passemos a considerá-lo. E mais, para $\frac{d}{c} \neq 0$, utilizando a homotetia $H: \mathbb{C} \longrightarrow \mathbb{C}$ dada por $H(x)=\frac{d}{c} x$, podemos considerar as transformações de Möbius na forma

$$
\phi(x ; a, b)=\frac{a x+b}{x+1}
$$

com $a, b \in \mathbb{R}$, para $x \neq-1$, que são as que servem às nossas necessidades, como veremos adiante. Oportunamente, observa-se que, se $c \cdot d>0$, dado $P \in \mathbb{R}[x]$, temos que $V(P \circ H)=V(P)$.

Na sequência, por um momento, consideramos $0<$ $a<b$. Sendo assim, $\phi$ transforma o intervalo $[0,+\infty)$ em $(a, b]$, e sua inversa é dada por

$$
\psi(x)=\psi(x ; a, b)=\frac{x-b}{a-x} .
$$

Observemos que $\phi$ é um difeomorfismo analítico.

Dado $P \in \mathbb{R}[x]$, denotaremos por $\left.P\right|_{(a, b]}$ o numerador da função racional $P \circ \phi$, onde $\phi=\left.\phi\right|_{[0,+\infty)}$. De fato,

$$
\left.P\right|_{(a, b]}=(x+1)^{n} \cdot P\left(\frac{a x+b}{x+1}\right),
$$

onde $n$ é o grau de $P$. Segue-se, imediatamente da regra de sinais de Descartes, que se $V\left(\left.P\right|_{(a, b]}\right)=0$ ou $V\left(\left.P\right|_{(a, b]}\right)=1$, então $P$ não tem zeros ou tem um único zero, respectivamente, no intervalo $(a, b]$. Isto mostra-nos a utilidade de $\phi$, possibilitando-nos obter informações sobre os zeros de $P$ em $(a, b]$, das informações obtidas da aplicação direta da regra de sinais de Descartes à $\left.P\right|_{(a, b]}$. Agora, vejamos como $\phi$ trabalha. Antes disso, observamos, como consequência do teorema fundamental da Álgebra, que todo polinômio $P \in \mathbb{R}[x]$ fatora-se completamente como um produto de fatores da forma $x-\alpha$, com $\alpha \in \mathbb{R}$, que chamamos de lineares, com fatores da forma $[x-(\rho+i \sigma)][x-(\rho-i \sigma)]=x^{2}-2 \rho x+\rho^{2}+\sigma^{2}$, $\operatorname{com} \rho, \sigma \in \mathbb{R}$, que chamamos quadráticos. Estas últimas formas são consequências do fato de que os zeros complexos de $P$ aparecem aos pares, já que se $\rho+i \sigma$ é um zero, $\rho-i \sigma$ também o é. Começemos com os fatores 
lineares, digamos, $P(x)=x-\alpha, \operatorname{com} \alpha \in \mathbb{R}$. Sendo assim,

$$
\left.P\right|_{(a, b]}=(a-\alpha)[x-\psi(\alpha)] .
$$

Disto, segue-se que se $\alpha \in(a, b), V\left(\left.P\right|_{(a, b)}\right)=1$, já que $\psi(\alpha)>0$. E se $\alpha \notin(a, b), V\left(\left.P\right|_{(a, b)}\right)=0$, já que $\psi(\alpha)<0$.

Passemos aos fatores quadráticos, digamos, $P(x)=$ $(x-\alpha)(x-\bar{\alpha}), \operatorname{com} \alpha \in \mathbb{C}$. Neste caso,

$$
\left.P\right|_{(a, b]}=|a-\alpha|^{2}\left[x^{2}-2 R\left(\frac{\alpha-b}{a-\alpha}\right) x+\left|\frac{\alpha-b}{a-\alpha}\right|^{2}\right],
$$

onde $R(\cdot)$ indica a parte real de um número complexo. Observemos que

$$
R\left(\frac{\alpha-b}{a-\alpha}\right)=\frac{(R(\alpha)-a)(b-R(\alpha))-I(\alpha)^{2}}{(a-R(\alpha))^{2}+I(\alpha)^{2}}
$$

onde $I(\cdot)$ indica a parte imaginária de um número complexo. Como

$$
\begin{aligned}
(R(\alpha)-a)(b-R(\alpha))-I(\alpha)^{2} & =-\left[\left(R(\alpha)-\frac{a+b}{2}\right)^{2}\right. \\
& \left.+\quad I(\alpha)^{2}-\left(\frac{b-a}{2}\right)^{2}\right],
\end{aligned}
$$

temos que $\alpha$ está no exterior ou sobre o círculo

$$
\left|z-\frac{a+b}{2}\right|=\frac{b-a}{2}
$$

se, e somente se, $V\left(\left.P\right|_{(a, b]}\right)=0$.

Existem outras condições suficientes que nos permitem concluir que

$$
(R(\alpha)-a)(b-R(\alpha))-I(\alpha)^{2}<0 .
$$

Imediatamente, vê-se que uma destas condições é que $\alpha$ não tenha parte real em $(a, b)$, isto é, $R(\alpha) \notin(a, b)$. Uma outra condição é que

$$
|b-a|<\Delta:=\min _{0 \leq i<j \leq n-1}\left|x_{j}-x_{i}\right|
$$

onde $x_{0}, x_{1}, x_{2}, \ldots, x_{n-1}$ são os $n$ zeros de $P \in \mathbb{R}[x]$. De fato, dado $\rho+i \sigma$, $\operatorname{com} \rho, \sigma \in \mathbb{R}$, um zero de $P$, temos que $\Delta<2|\sigma|$. Como $(\rho-a)(b-\rho)=-\left[\rho^{2}-(a+b) \rho+a b\right]$, temos que o seu valor máximo é atingido em $\frac{1}{2}(a+b)$, $\mathrm{e}$ vale $\frac{1}{4}(a-b)^{2}$. Desta forma,

$$
(\rho-a)(b-\rho) \leq \frac{1}{4}(a-b)^{2}<\frac{1}{4} \Delta^{2}<\sigma^{2} .
$$

$\mathrm{O}$ que nos mostra que $(\rho-a)(b-\rho)-\sigma^{2}<0$. Além disso, observamos que a condição $b-a<\Delta$ implica que no máximo um zero real de $P$ está no disco

$$
\left\{z \in \mathbb{C}:\left|z-\frac{a+b}{2}\right|<\frac{b-a}{2}\right\} \text {. }
$$

Uma consequência imediata desses fatos é que
Proposição 2.1. Se os zeros de $P \in \mathbb{R}[x]$ têm parte real não-positiva, então $V(P)=0$.

E também, concluímos que, dado $P \in \mathbb{R}[x],\left.P\right|_{(a, b]}$ tem a seguinte fatoração

$$
\begin{array}{r}
P(a) \cdot \ldots \cdot[x-\psi(\alpha)] \cdot \ldots \cdot\left[x^{2}-2 R(\psi(\alpha))\right. \\
\left.+|R(\psi(\alpha))|^{2}\right] \cdot \ldots
\end{array}
$$

As considerações acima, leva-nos a um teorema, devido a Lagrange Lagrange (1808), que serviu como inspiração ao resultado de Vincent (1836), e é, de uma certa forma, uma recíproca à regra de sinais de Descartes. Aqui, adotamos o enunciado clássico de Lagrange. Como veremos na demonstração, as hipóteses no enunciado podem ser substituídas pelas condições mencionadas acima.

Teorema 2.4. Seja $P \in \mathbb{R}[x]$ de grau n. Suponha que $P$ tem um único zero simples $x_{0} \mathrm{em}(a, b)$, e que nenhum zero complexo de $P$ tem parte real neste intervalo. Se a é escolhido suficientemente próximo a $x_{0}$, então $V\left(\left.P\right|_{(a, b]}\right)=1$, onde $(t, s)=(0,1)$.

Demonstração. Consideremos $x_{1}, x_{2}, \ldots, x_{n-1}$ os outros zeros de $P$. Observemos que cada $x_{j}$ é transformado por $\psi$ em

$$
\frac{x_{j}-b}{a-x_{j}} .
$$

Sendo assim, $x_{j} \in(a, b)$ se, e somente se, $\psi\left(x_{j}\right)=: \xi_{j}>0$. Neste caso, $x_{j}=x_{0}$.

Como vimos acima, o polinômio $\left.P\right|_{(a, b]}$ é da forma

$K\left(x-\xi_{0}\right) \cdot \ldots \cdot\left(x-\xi_{j}\right) \cdot \ldots \cdot\left(x^{2}-2 R\left(\xi_{j}\right) x+\left|R\left(\xi_{j}\right)\right|^{2}\right) \cdot$

para $j=1,2, \ldots, n-1$, onde $K$ é uma certa constante real. Como, por hipótese, $R\left(x_{j}\right) \notin(a, b)$, para $j=1,2, \ldots, n-$ 1 , temos que os coeficientes do polinômio

$$
\cdots\left(x-\xi_{j}\right) \cdot \ldots \cdot\left(x^{2}-2 R\left(\xi_{j}\right) x+\left|R\left(\xi_{j}\right)\right|^{2}\right) \cdot \ldots
$$

são positivos. Escrevendo este polinômio como

$$
b_{n-1} x^{n-1}+b_{n-2} x^{n-2}+\ldots+b_{1} x+b_{0},
$$

onde $b_{i}>0$, para $i=0,1,2, \ldots, n-1$, temos que

$$
\begin{array}{r}
\left.P\right|_{(a, b]}=K\left[b_{n-1} x^{n}+\left(b_{n-2}-\xi_{0} b_{n-1}\right) x^{n-1}+\ldots+\right. \\
\left.+\left(b_{0}-\xi_{0} b_{1}\right) x-\xi_{0} b_{0}\right] .
\end{array}
$$

Observando que $\xi_{0}=\frac{x_{0}-a}{b-x_{0}}$, com $a<b$, e permitindo-se variar o parâmetro $a$, temos que existe $\delta>0$ tal que

$$
\xi_{0}<\min _{1 \leq k \leq n-1} \frac{b_{k-1}}{b_{k}}
$$


para todo $a \in\left(x_{0}-\delta, x_{0}\right)$. Sendo assim, $b_{k-1}-\xi_{0} b_{k}>0$, para $k=1,2, \ldots, n-1$. Logo, $V\left(\left.P\right|_{(a, b]}\right)=1$, com $(t, s)=(0,1)$.

Um fato imediato da demonstração acima, é que, nas condições do teorema, para $a$ suficientemente próximo de $x_{0}$, mesmo que o polinômio original, digamos, $P$, seja incompleto, isto é, se algum dos seus coeficientes é nulo, o transformado, $\left.P\right|_{(a, b]}$, é completo.

\section{A vesão clássica}

Nesta seção, apresentaremos duas versões clássicas do teorema de Vincent. Aqui, clássica significa: em termos de frações contínuas. A primeira versão é a encontrada em Akritas (1981). A segunda versão é a de Alesina e Galuzzi (1998), que explora as propriedades das transformações de Möbius. Na demonstração original de Vincent (1836), o argumento principal encontra-se na demonstração do teorema 2.4. Nesta primeira versão, aparece uma estimativa que depende do grau do polinômio. Passemos a primeira versão.

Teorema 3.1. (Vincent-Uspensky-Akritas) Sejam $P \in \mathbb{R}[x]$, de grau $n>1$ e sem zeros múltiplos, e $\Delta>0$ a menor distância entre quaisquer dois zeros de P. Considere $m o$ menor indice tal que

$$
F_{m-1} \frac{\Delta}{2}>1 \text { e } F_{m-1} F_{m} \Delta>1+\frac{1}{\epsilon_{n}},
$$

onde $F_{k}$ é o k-ésimo termo da sequência de Fibonacci

$$
1,1,2,3,5,8,13,21, \ldots
$$

$e$

$$
\epsilon_{n}=\left(1+\frac{1}{n}\right)^{\frac{1}{n-1}}-1 .
$$

Então, as transformações sucessivas um algoritmo de tempo computacional polinomial, diferentemente do original de Vincent, que tem tempo computacional exponencial, para a obtenção dos $a_{i}{ }^{\prime}$, conforme Akritas (1979).

O próximo resultado, que é crucial na demonstração da próxima versão clássica do teorema de Vincent, é, como veremos, por si só muito interessante. Uma demonstração pode ser encontrada em Alesina e Galuzzi (1998). Cabe observar que o mesmo resultado foi obtido por Ostrowski (1950), conforme lema 3, página 705.

Proposição 3.1. Se $P \in \mathbb{R}[x]$ tem um zero positivo simples, e todos os demais zeros, possivelmente múltiplos, no setor

$$
S_{\sqrt{3}}=\left\{x=\rho+i \sigma: \rho<0 e \sigma^{2} \leq 3 \rho^{2}\right\},
$$

então $V(P)=1$.

Passemos a próxima versão clássica do teorema de Vincent. Nesta versão, a estimativa análoga àquela da primeira versão independe do grau do polinômio. Em Ostrowski (1950), tem-se uma estimativa menor do que 3 , porém, maior do que $\frac{2}{\sqrt{3}}$, qual seja, $\sqrt{3}$. Uma demonstração do teorema abaixo pode ser vista em Alesina e Galuzzi (1998); Ostrowski (1950).

Teorema 3.2. (Vincent-Alesina-Galuzzi) Sejam $P \in \mathbb{R}[x]$, de grau $n>1$ e sem raízes múltiplas, e $\Delta>0$ a menor distância entre quaisquer dois zeros de P. Considere $\frac{p_{k}}{q_{k}}$ como o k-ésimo convergente da fração contínua

$$
a_{0}+\frac{1}{a_{2}+\frac{1}{a_{3}+\ddots}},
$$

onde $a_{i}$ é um inteiro positivo, para cada $i \geq 1$, e $a_{0} \geq 0$. Se $h$ é tal que

$$
F_{h-1} F_{h} \Delta>\frac{2}{\sqrt{3}}
$$

onde $F_{k}$ é o k-ésimo termo da sequência de Fibonacci

$$
1,1,2,3,5,8,13,21, \ldots,
$$

com $a_{1}, a_{2}, a_{3}, \ldots, a_{m}$ inteiros positivos arbitrários, transformam $P$ em $\tilde{P}$ tal que $V(\tilde{P}) \leq 1$, onde $\tilde{P}$ é o numerador da função racional obtida ao final das iterações acima.

É fácil ver que, para todo $n \geq 2$, temos que $\epsilon_{n} \leq \frac{1}{2}$. E então,

$$
F_{m-1} F_{m} \Delta>1+\frac{1}{\epsilon_{n}} \geq 3 .
$$

É importante salientar que o interesse, em especial, de Akritas é descrever um método eficiente para separar zeros de um polinômio, e assim, determinar aproximações ótimas para estes zeros. Para tanto, constroí

\section{A versão geométrica}

Inicialmente, relembremos, ver seção 1, que Ostrowski (1950) exibiu uma demonstração do teorema de Vincent, distinta daquelas dadas em Akritas (1981); Uspensky (1948), e com alguma semelhança àquela dada em Alesina e Galuzzi (1998), tanto que em Alesina e Galuzzi (1999), os autores resolveram publicar uma nota defendendo a independência dos seus resultados. A seguir, enunciamos os resultados de Ostrowski que utilizaremos. O primeiro, possivelmente inspirado pelo teorema 2.4, é o seguinte 
Proposição 4.1. Seja $P(x)=b_{n} x^{n}+b_{n-1} x^{n-1}+\ldots+$ $b_{1} x+b_{0}$, com $b_{j}>0$, para $j=0,1,2, \ldots, n$. Uma condição necessária e suficiente para que, dado $\alpha>0, V((x-\alpha) P)=1$ é que $b_{k}^{2} \geq b_{k-1} b_{k+1}$, para cada $k=1,2, \ldots, n-1$.

Relembramos que, ver o final da seção 2, a demonstração do teorema 2.4 mostra-nos que mesmo quando o polinômio considerado é incompleto, o seu transformado é completo. Da leitura da demonstração da proposição acima, como vista em Ostrowski (1950), notamos que se todas as $n-1$ desigualdades acima são estritas, então, mesmo que o polinômio original seja incompleto, o polinômio transformado $\left.P\right|_{(a, b]}$ terá todos os coeficientes não-nulos, exceto possivelmente para um deles.

O segundo resultado, cuja versão mais geral, para séries normais, pode ser vista em Ostrowski (1939), é o seguinte

Proposição 4.2. Sejam $P, Q \in \mathbb{R}[x]$, com coeficientes positivos, que satisfazem a condição da proposição acima. Então, $P \cdot Q$, também, satisfaz tal condição.

A seguir, o interesse é explorar as propriedades geométricas das transformações de Möbius. Considerando a aplicação $\psi$, temos que, dado $\alpha \in \mathbb{C}$,

$\psi(\alpha)=\frac{1}{|\alpha-a|^{2}}\left[-\left|\alpha-\frac{a+b}{2}\right|^{2}+\left(\frac{b-a}{2}\right)^{2}+i(a-b) I(\alpha)\right]$.

Disto segue-se que

1. Se $\alpha$ está sobre o círculo

$$
\Gamma:\left|z-\frac{a+b}{2}\right|=\frac{b-a}{2},
$$

então

$$
\psi(\alpha)=i \frac{(a-b) I(\alpha)}{|\alpha-a|^{2}} .
$$

Ou seja, os pontos sobre o círculo são levados em pontos sobre o eixo imaginário.

2. Se $\alpha$ é um ponto exterior ao ou sobre o círculo $\Gamma$, então $R(\psi(\alpha)) \leq 0$. Desta forma, se um ponto é interior ao círculo $\Gamma$, então $R(\psi(\alpha))>0$.

3. As retas $I(\cdot)= \pm s R(\cdot)$, para cada $s \in \mathbb{R} \backslash\{0\}$, são as imagens através de $\psi$ dos círculos, denotados por $\Gamma^{ \pm}$, centrados em

$$
c^{ \pm}=\frac{a+b}{2} \pm i \frac{b-a}{2 s} \text { de raio } r=\frac{b-a}{2} \sqrt{1+\frac{1}{s^{2}}} .
$$

De fato, desenvolvendo

$$
\left|\alpha-\left(\frac{a+b}{2} \pm i \frac{b-a}{2 s}\right)\right|=\frac{b-a}{2} \sqrt{1+\frac{1}{s^{2}}}
$$

chegamos a

$$
\pm s\left[(R(\alpha)-a)(b-R(\alpha))-I(\alpha)^{2}\right]=(b-a) I(\alpha) .
$$

4. Seja $s>0$. A imagem dos pontos exteriores à $\Gamma^{+} \cup \Gamma^{-}$por $\psi$ é o setor

$$
S_{s}=\{z \in \mathbb{C}: R(z)<0 e|I(z)|<s|R(z)|\} .
$$

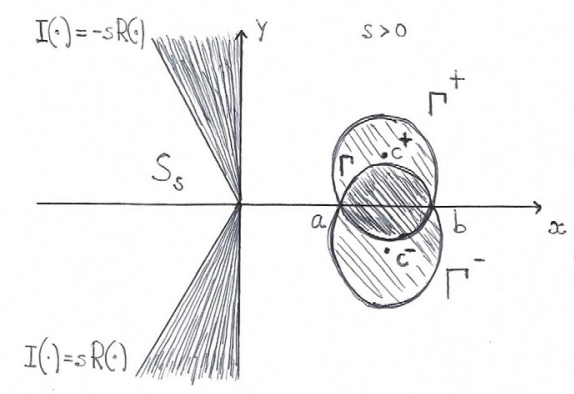

A seguir, vemos a versão geométrica do teorema de Vincent, como encontramos em Alesina e Galuzzi (2000). Mas, diferentemente de Alesina e Galuzzi, na demonstração optamos por usar os resultados em Ostrowski (1950).

Teorema 4.1. Seja $P \in \mathbb{R}[x]$, de grau $n>1$ e sem zeros múltiplos. É possível determinar $\delta>0$ tal que, para todos $a, b>0$, digamos, com $a<b e b-a<\delta, V\left(\left.P\right|_{(a, b]}\right) \leq 1$. E mais,

$$
\begin{gathered}
V\left(\left.P\right|_{(a, b]}\right)=1 \text { se, e somente se, } P \text { tem um único zero } \\
\text { simples em }(a, b) .
\end{gathered}
$$

Demonstração. Basta, dado $0<s \leq \sqrt{3}$, tomarmos $\delta=\frac{s}{\sqrt{1+s^{2}}} \Delta$. Sendo assim, como

$$
2 r=(b-a) \sqrt{1+\frac{1}{s^{2}}}
$$

temos que $2 r<\Delta$. Disto, segue-se que

- ou todos os zeros de $P$ são exteriores ao círculo $\Gamma$. E assim, por 2 e pela proposição $2.1, V\left(\left.P\right|_{(a, b]}\right)=$ 0 .

- ou somente um zero de $P$, necessariamente real, é interior à $\Gamma$, e os demais, por 4 , são levados por $\psi$ em $S_{s}$.

Para o segundo caso, indicando por $\xi_{0}=\psi\left(x_{0}\right)$, onde $x_{0}$ é o único zero de $P$ em $(a, b)$, temos que $\left.P\right|_{(a, b]}$ é um produto da forma $K\left(\xi-\xi_{0}\right) \cdot Q(\xi)$, para um certo $K \in \mathbb{R}$, onde $Q$ é um produto de fatores lineares da forma $\xi-\eta$, com $\eta<0$, e quadráticos da forma $\xi^{2}-2 \eta \xi+\eta^{2}+\mu^{2}$, com $\eta<0$. Como $\eta \pm i \mu \in S_{s}$, temos que $\mu^{2}<s^{2} \eta^{2}$. Logo,

$$
\eta^{2}+\mu^{2}<\left(1+s^{2}\right) \eta^{2} \leq(2 \eta)^{2},
$$


já que $s \leq \sqrt{3}$. Desta forma, os fatores quadráticos de $Q$ satisfazem a condição de Ostrowski, conforme proposição 4.1. Além disso, como $Q$ é um produto de polinômios que satisfazem a condição de Ostrowski, temos, pela proposição 4.2 , que $Q$ também satisfaz esta condição. Novamente, pela proposição 4.1, temos que $V\left(\left.P\right|_{(a, b]}\right)=1$, com a adição de que o transformado é um polinômio completo. E assim, os dois casos mostram que $V\left(\left.P\right|_{(a, b]}\right) \leq 1$. A segunda parte do teorema é evidente.

Observação 4.1. O teorema 4.1 mostra-nos que, dado $P \in \mathbb{R}[x]$, cujo grau é maior do que 1 , sem zeros múltipos, existe uma partição da reta real, por intervalos de diâmetros menores do que um certo $\delta>0$, tal que, em cada um destes intervalos, encontra-se no máximo um zero de $P$. É claro que, dentre estes intervalos, existem no máximo a quantidade correspondente ao grau de $P$ que tem exatamente uma variação de sinal.

Para obtermos o resultado como em Alesina e Galuzzi (2000), tomamos $s=\sqrt{3}$, e usamos (4). É claro, que a demostração que damos amplia, significativamente, o campo de valoração de $s$. Geometricamente, isto significa que podemos ampliar o sector $S_{s}$, tornando-o tão próximo do setor $\left\{\alpha \in \mathbb{C}: \frac{\pi}{2} \leq \arg (\alpha) \leq \frac{3 \pi}{2}\right\}$, tanto quanto quisermos. Mas, isto significa que $\delta \leq \frac{\sqrt{3}}{2} \Delta$. E assim, o comprimento do intervalo de extremos $a$ e $b$ é menor do que $\frac{\sqrt{3}}{2} \Delta$.

Observemos que, a príncipio, poderíamos pensar num intervalo $(a, b)$ com extremos quaisquer, não necessariamente positivos. Bem, se os extremos fossem negativos, usaríamos a mudança $x \mapsto-x$, e procederíamos como no teorema. Agora, se os extremos fossem de sinais contrários, digamos, com $a<0<b$, dividiríamos este intervalo em dois subintervalos, no caso, $(a, 0)$ e $(0, b)$, e procederíamos como antes em relação ao intervalo $(a, 0)$. A verificação de que 0 é um zero de um polinômio $P$ é direta. Desta forma, basta considerarmos o caso prescrito pelo teorema.

Como uma consequência do teorema 4.1, temos o seguinte:

Corolário 4.2. Seja $P \in \mathbb{R}[x]$, de grau $n>1$. Então, $x_{0}$ não é um zero de $P$ se, e somente se, existem $a, b \in \mathbb{R}$, com $a<b$ e $x_{0} \in(a, b)$, tais que

$$
V\left(\left.P\right|_{(a, b]}\right)=0 .
$$

Além disso, podemos escolher a tal que os coeficientes de

$$
\tilde{P}(u)=(u+1)^{m} \cdot P\left(\frac{a u+b}{u+1}\right)
$$

tenham o mesmo sinal de $P(a)$.

Observação 4.2. A última parte do corolário, por exemplo, segue do seguinte argumento:

$$
\begin{aligned}
\tilde{P}(u)= & (u+1)^{m} \cdot P\left(a+\frac{b-a}{u+1}\right) \\
= & P(a)(u+1)^{m}+\frac{P^{\prime}(a)}{1 !}(b-a)(u+1)^{m-1}+ \\
& \cdots+\frac{P^{m}(a)}{m !}(b-a)^{m} \\
= & P(a) u^{m}+\left[\left(\begin{array}{c}
m \\
m-1
\end{array}\right) P(a)+\frac{P^{\prime}(a)}{1 !}(b-a)\right] \\
& u^{m-1}+\cdots+\left[\left(\begin{array}{c}
m \\
0
\end{array}\right) P(a)+\left(\begin{array}{c}
m-1 \\
0
\end{array}\right) \frac{P^{\prime}(a)}{1 !}\right. \\
& \left.(b-a)+\cdots+\frac{P^{m}(a)}{m !}(b-a)^{m}\right] .
\end{aligned}
$$

\section{Conclusões}

A versão apresentada em Alesina e Galuzzi (2000), através de computação exata e simbólica, possibilita-nos testar diretamente se um dado intervalo contém zeros de um determinado polinômio real numa variável. E mais, se existe um zero simples deste polinômio no tal intervalo, podemos obter subintervalos do intervalo inicial cujo diâmetro é tão pequeno quanto se queira ao redor do zero. A demonstração que propomos para esta versão baseia-se nos resultados encontrados em Ostrowski (1950), que é diferente da demonstração dada em Alesina e Galuzzi (2000), apesar de utilizarmos muitas das suas idéias preliminares. Para os nossos interesses em pesquisa, a principal vantagem da versão geométrica do teorema de Vincent, juntamente com a regra de sinais de Descartes e as transformações de Möbius, é a que mencionamos acima, ou seja, a possibilidade de realizarmos computação exata e simbólica, como pode ser verificada na abordagem dada em Barros e Leandro $(2011,2014)$ e em Leandro $(2003,2006)$ para estudar finitude e enumeração de classes de configurações centrais no problema de $n$ corpos. E mais, esta versão permite-nos estender o teorema para várias variáveis, que é o trabalho que pretendemos concluir em breve, o que nos garantirá explicar o bom funcionamento do método mencionado.

\section{Referências}

Ahlfors, L. V. (1949). Complex Analysis: an introduction to the theory of analytic functions of one complex variable. McGraw-Hill.

Akritas, A. G. (1978). A correction on a theorem by uspensky. Bull Soc Math Greece, (19), 278-285. 
Akritas, A. G. (1979). On the solution of polynomial equations using continued fractions. Information Processing Letters, 9(4), 182-184.

Akritas, A. G. (1981). Vincent's forgotten theorem, its extension and application. Comp $\mathcal{E}$ Maths with Appls, (7), 309-317.

Akritas, A. G. (1986). There is no "uspensky"method. In: Proceedings of the 1986 Symposium on Simbolic and Algebraic Computation, Waterloo, Ontario, Canada, pp. 88-90.

Akritas, A. G., Danielopoulos, S. D. (1978). On the forgotten theorem of mr. vincent. Historia Math, (5), 427-435.

Alesina, A., Galuzzi, M. (1998). A new proof of vincent's theorem. L'Enseignement Matématique, 44, 219-256.

Alesina, A., Galuzzi, M. (1999). Addendum to the paper "a new proof of vincent's theorem". L'Enseignement Matématique.

Alesina, A., Galuzzi, M. (2000). Vincent's theorem from a modern point of view. Categorical Studies in Italy, Rendiconti del Circolo Matematico de Palermo, (Serie II, 64), 179-191.

Barros, J. F., Leandro, E. S. G. (2011). The set of degenerate central configurations in the planar restricted fourbody problem. SIAM Journal of Mathematical Analysis, (43), 634-661.

Barros, J. F., Leandro, E. S. G. (2014). Bifurcations and enumeration of classes of relative equilibria in the planar restricted four-body problem. SIAM Journal of Mathematical Analysis, (46-2), 1185-1203.

Descartes, R. (1637). La geometrie in discours de la methode. Dover.

Eigenwillig, A. (2008). Real root isolation for exact and approximate polynomials using descartes's rule of signs. Tese de Doutorado, Saarland University.

Lagrange, J. P. (1808). Traite de la resolution des equations numeriques de tous les degres, avec des notes sur plusieurs points de la theorie des equations algebriques. chez Courcier.

Leandro, E. S. G. (2003). Finiteness and bifurcations of some symetrical classes of central configurations. Arch Rational Mech Anal.

Leandro, E. S. G. (2006). On the central configurations of the planar restricted four-body problem. Journal of Differential Equations, (226), 323-351.

Obreschkoff, N. (1963). Verteilung und berechnung der nullstellen reeller polynome. VEB Deustscher Verlag der Wissenschaften.
Obreschkoff, N. (2003). Zeros of polynomials. Marin Drinov.

Ostrowski, A. M. (1939). Note sur les produits de series normales. Bulletin de la Société Royale des Sciences de Liége, pp. 458-468.

Ostrowski, A. M. (1950). Note on vincent's theorem. The Annals of Mathematics, 52(3), 702-707.

Uspensky, J. V. (1948). Theory of equations. McGraw-Hill.

Vincent, A. J. (1836). Sur la resolution des equations numeriques. J Math Pures Appl, (1), 341-372.

Wang, X. (2004). A simple proof of descartes's rule of signs. The American Mathematical Monthly, (111), 525526. 